\title{
CARACTERÍSTICAS DAS CRISES EPILÉPTICAS APÓS ACIDENTE VASCULAR CEREBRAL ISQUÊMICO
}

\author{
MARCIA MAIUMI FUKUUIMA*, JOSÉ OSMAR CARDEAL **
}

\begin{abstract}
RESUMO - Os infartos cerebrais constituem uma das mais importantes causas de epilepsia de início tardio. Foram estudados 35 pacientes que apresentaram crises epiléptica com início 24 horas após a instalação do acidente vascular cerebral isquêmico (AVCI) e que tiveram pelo menos 1 recorrência da crise, a fim de definir as principais características dessas crises e correlacioná-las aos principais achados clínicos e laboratoriais. $\mathrm{O}$ tempo entre a instalação do AVCI e a primeira crise foi de 3 a 1650 dias, com predomínio (89\%) de crises de instalação tardia (>14 dias); houve um pico de frequência no periodo de 6 a 12 meses após a instalação do AVC. Crises parciais foram mais frequentes (31/35) do que crises primariamente generalizadas, e estado de mal epiléptico só ocorreu em 3 casos. A maioria (30/35) apresentou crises pouco frequentes ( $\leq 1 \mathrm{crise} / \mathrm{mês}$ ). Não houve associação entre tipo de crise e intervalo de tempo entre ela e a instalação do AVCI, nem com a frequência. A alteração eletrencefalográfica mais frequente foi alentecimento focal da atividade elétrica cerebral.Todos os pacientes obtiveram controle das crises com monoterapia.
\end{abstract}

PALAVRAS-CHAVE: infarto cerebral, crise epiléptica, epilepsia tardia.

\section{Characteristics of epileptic seizures after ischemic stroke}

ABSTRACT - Cerebral infarctions are one; of the most important causes of late onset epilepsy. We have studied 35 patients who presented epileptic seizures after ischemic stroke. All of them had the first seizure at least 24 hours after the stroke, and they had at least one recurrence of seizure. The objective was to determine the main characteristics of these seizures and to correlate them to clinical and laboratorial findings. The interval between the stroke and the first seizure was 3 to 1650 days. Late onset seizures (>14 days) were present in $89 \%$, they occurred predominantly 6 to 12 months after stroke. Partial seizures (31/35) were more frequent than generalized ones. Status epilepticus occurred in only 3 cases. Most of the patients (30/35) had occasional seizures $(\leq 1$ seizure monthly). There were no association between seizure type and the time interval between the stroke and the first seizure, neither with the seizure frequency. The most frequent EEG finding was focal slowing of cerebral activity. Pharmacological control was easily obtained. No patient needed more than one drug for seizure control.

KEY WORS: cerebral infarction, late onset epilepsy, epileptic seizure.

Estima-se que somente $30 \%$ das pessoas que sofrem acidente vascular cerebral (AVC) recuperem todas as funçōes afetadas. As principais consequências dos AVC são sequela motora e de linguagem. Porém, várias outras complicaçōes podem ocorrer, entre elas, as crises epilépticas.

Epilepsia é conceituada como uma síndrome caracterizada por recorrência de crises epilépticas, associada a diferentes etiologias, mas com mecanismo fisiopatológico comum ${ }^{6}$. Um dos critérios

Estudo realizado no Setor de Doenças Neurovasculares da Disciplina de Neurologia da Escola Paulista de Medicina (EPM) da Universidade Federal de São Paulo (UNIFESP), baseado na Tese de Mestrado "Crises epilépticas após acidente vascular cerebral por infarto cerebral: estudo de 35 pacientes"21.22: *Neurologista na EPM - UNIFESP; **Professor Adjunto da Disciplina de Neurologia da EPM - UNIFESP. Aceite: 7-julho-1997.

Dra. Marcia Maiumi Fukujima - Universidade Federal de São Paulo, Disciplina de Neurologia - Rua Botucatu 740 - 04023-900 São Paulo SP - Brasil. FAX 011575 5240. E-mail: Maiumi@sti.com.br ou maiumi@sunnepi.epm.br. 
para classificação das síndromes epilépticas baseia-se na idade de início das crises. Este critério é importante para estabelecer a etiologia das epilepsias ${ }^{12}$. Estima-se que apenas 20 a $25 \%$ dos pacientes com epilepsia tenham a primeira crise epiléptica após 25 anos de idade ${ }^{16,35}$. As crises epilépticas da idade adulta, também chamadas de crises epilépticas de início tardio, são bastante estudadas, porém a idade para a crise ser considerada de início tardio nāo é bem estabelecida na literatura.

Os AVC constituem uma das causas mais importantes de epilepsia de início tardio, sendo observados em $14 \%$ dos indivíduos epilépticos com idade superior a 25 anos $^{13}$ e em cerca de 30 a $45 \%$ dos indivíduos epilépticos com mais que 60 anos ${ }^{1,19,26,33.50}$. Os traumatismos cranioencefálicos têm sido considerados uma das principais causas de epilepsia de início tardio, constituindo a principal etiologia de crises epilépticas em indivíduos na faixa etária de 20 a 40 anos; o mesmo ocorre com os AVC nos indivíduos com mais que 50 anos de idade ${ }^{15,20,28,39,41,44,47,53}$. As etiologias das crises epilépticas de início tardio variam de açrło com a geografia e com critérios diagnósticos utilizados. Neurocisticercose, por exemplo, representa a etiologia em $45 \%$ dos casos de epilepsia em países onde sua prevalência é alta ${ }^{46}$. No nosso meio observa-se que $14 \%$ das crises epilépticas de início tardio têm como causa a doença vascular cerebral, só sendo menos frequente que a neurocisticercose (19\%) e que os casos idiopáticos $(47 \%)^{52}$. Em estudo populacional americano recente, observou-se 18 casos de epilepsia em 639 infartos cerebrais acompanhados por longo periodo de tempo, tendo sido calculado que o infarto cerebral eleva em 17 vezes o risco de epilepsia comparado à população geral ${ }^{44}$.

A frequência de crises epilépticas como complicação tardia de AVC, isto é, após 14 dias da sua instalação, varia de 3 a 19\% $17,32,37,38,40,44$, e a frequência de crises epilépticas na instalação do AVC, ou seja, nos primeiros 14 dias, varia de 3,3 a 4,3\% 32,42 . Estima-se um risco de $6 \%$ para crises epilépticas após AVC, porém com declínio de $0,4 \%$ ao ano ${ }^{4 \%}$, embora estudos apontem que apenas 1 a $2 \%$ dos AVC isquêmicos (AVCI), especifīcamente, evoluam com crises epilépticas ${ }^{17,36}$. Devido à alta prevalência de AVC (200 por 100000 habitantes) em relação à de epilepsia (30 a 50 por 100000 habitantes), AVC continua sendo uma das mais importantes causas conhecidas de epilepsia, principalmente em indivíduos idosos.

Alguns autores postulam que a epilepsia que precede o AVC seja, em geral, desencadeada por outros fatores diferentes da doença vascular cerebral, tais como insuficiência renal, hipertensão arterial, edema cerebral, hipertensāo intracraniana ou distúrbios hemodinâmicos, alguns dos quais considerados fatores de risco para o próprio $\mathrm{AVC}^{14}$. As crises epilépticas da fase aguda ocorrem devido a alteraçōes metabólicas citotóxicas consequentes da lesāo cerebral, que podem induzir crises epilépticas por si só, ou podem ocorrer em um substrato adjacente favorável, como por exemplo, um AVC antigo ou predisposição genética. A recorrência de crises epilépticas surge quando o AVC causa uma lesāo estrutural ${ }^{8,2 y, 30,51}$.

Este estudo tem o objetivo de analisar as principais características das crises epilépticas que ocorrem após 24 horas de instalação do AVCI, tais como o tipo e frequência das crises, tempo de ocorrência em relação ao AVCI e correlacioná-las aos achados clínicos e laboratoriais.

\section{CASUÍSTICA E MÉTOdOS}

Foram estudados 35 pacientes atendidos no período de 1990 a 1992 que apresentaram crises epilépticas com início 24 horas após a instalação do AVCI e com pelo menos uma recorrência da crise, sendo 20 homens e 15 mulheres, com idade entre 32 e 85 anos. Optamos por estudar as crises de ocorrência após 24 horas da instalação do $\mathrm{AVCI}$ porque as crises que ocorrem nesse periodo inicial são atribuídas a fatores outros que não o AVCI (distúrbios metabólicos, hemodinâmicos, abstinência a drogas e álcool, por exemplo).

Todos os diagnósticos de AVCI foram feitos por quadro clínico e tomográfico e classificados segundo a Classificação de Doenças Cerebrovasculares III, de 1990, do National Institute of Neurological Disorders and Stroke ${ }^{49}$. Foram excluídos pacientes: que desenvolveram síndrome de abstinência; submetidos a intervenções cirúrgicas intracranianas ou a cirurgias neurovasculares; com história de crises epilépticas prévias ao AVCI; que 
proporcionaram informaçōes insuficientes para o diagnóstico de crises epilépticas; com antecedente de encefalopatia por trauma cranioencefálico, anóxia, infeç̧̄̄es e tumor; com neurocisticercose; cujo exame de líquor cefalorraquidiano (LCR) ou tomografia computadorizada de crânio (TC) foi sugestivo de lesão hemorrágica, inflamatória, tumoral ou traumática; que tenham tido crises epilépticas apenas durante a instalação do AVC.

Todos os pacientes foram submetidos a anamese e exame clínico e neurológico. Nos antecedentes mórbidos pessoais, foi dado especial enfoque aos fatores de risco para doenças vasculares, tais como hipertensão arterial, diabetes melito, ataque isquêmico transitório (AIT), AVC pregresso, enxaqueca, uso de anticoncepcional oral, doença de Chagas comprovada sorologicamente, cardiopatia como possível causa de embolia cerebral, etilismo (que foi considerado positivo quando o paciente referiu uso de pelo menos uma dose de bebida alcoólica diariamente) e tabagismo (quando o paciente referiu uso de qualquer quantidade de cigarros diariamente).

O tipo de crise epiléptica foi classificado de acordo com a Classificação Internacional de Crises Epilépticas $^{11}$. Não nos preocupamos em classificar as epilepsias sindromicamente, por se tratar de epilepsia secundária a infarto cerebral em todos os casos.

Estado de mal epiléptico foi definido como crises epilépticas que se repetem com frequência suficiente para produzir uma condição neurológica fixa e duradoura durante pelo menos 30 minutos ou como uma série de duas ou mais crises epilépticas sem a recuperaçāo da consciência entre elas?

As crises epilépticas foram classificadas segundo a frequência em: pouco frequentes (uma ou menos crise por mês), e frequentes (mais que uma crise por mês). Segundo o período entre a instalação do AVCI e a primeira crise em: precoces ( 1 a 14 dias) e tardias (mais que 14 dias).

Para análise dos resultados foram aplicados os seguintes testes: 1 . Teste de Mann-Whitney, com a finalidade de comparar duas a duas, as variáveis estudadas. Este teste foi feito com aproximação à curva normal (z) quando o tamanho da amostra assim o exigiu ${ }^{43} ; 2$. Teste do quiquadrado para tabelas $2 \times 2$, com a finalidade de analisar as associaçōes entre as variáveis estudadas. Quando observadas as restriçōes de Cochran, foi aplicado o teste exato de Fisher ${ }^{43}$. O nível de significância foi fixado em 0,05 ou $5 \%(\alpha<0,05)$.

\section{RESULTADOS}

O tempo entre a instalação do AVCI e a primeira crise epiléptica variou 3 a 1650 dias, com média de 340,9 dias. Não houve diferença significante entre os sexos para o tempo entre o AVCI e a primeira crise epiléptica (teste de Mann-Whitney: Zcalculado=0,10; Zcrítico=1,96).

Crises de aparecimento precoce ( 1 a 14 dias) ocorreram em $11 \%$ dos pacientes e crises de aparecimento tardio ( $>14$ dias) em $89 \%$, sem diferença estatisticamente significante entre elas; sendo que $34 \%$ dos pacientes apresentaram crise no período de 15 a 180 dias; $54 \%$ após 180 dias, e somente $17 \%$ após 1 ano.

Crises parciais (31/35 pacientes) foram mais frequentes do que crises primariamente generalizadas (4/35 pacientes), tanto para homens quanto para mulheres, porém sem significância estatística. Dos pacientes com crises parciais, $11 / 18$ do sexo masculino e $8 / 13$ do sexo feminino apresentaram generalização subsequente. Apenas 1 dos 31 pacientes com crises parciais apresentou crise parcial complexa, todos os demais crises parciais simples motora.

Estado de mal epiléptico ocorreu em 3 pacientes. Apesar de só ter ocorrido no sexo masculino, a diferença nāo foi significante. Suas idades foram de 58,73 e 65 anos, respectivamente. No primeiro foi constatado somente antecedente de etilismo; no segundo, hipertensão arterial, AVC pregresso e tabagismo; e no terceiro, hipertensão arterial, AIT pregresso, etilismo e tabagismo. Os três evoluíram com crises epilépticas do tipo parcial e somente um com crises muito frequentes. $O$ tempo entre 0 AVCI e o episódio de mal epiléptico foi 44, 3 e 300 dias respectivamente, constituindo em todos os casos a primeira manifestaçăo epiléptica.

Quanto à frequência, predominaram crises pouco frequentes (30/35 paciente), isto é, uma crise ou menos por mês. 
Tabela 1. Frequência das crises epilépticas: baixa frequência ( $\leq 1$ crise/mês) e alta frequência (>Icrise/mês), segundo o tipo de crise parcial e generalizada e segundo o tempo de aparecimento da crise em relação ao AVCI: precoce (1-14 dias) e tardia (>14 dias). Resultado do teste exato de Fisher.

\begin{tabular}{lcccc}
\hline & \multicolumn{4}{c}{ Frequência das crises } \\
\cline { 2 - 5 } & $\begin{array}{l}\text { Baixa } \\
\mathrm{n}=30\end{array}$ & $\begin{array}{l}\text { Alta } \\
\mathrm{n}=5\end{array}$ & $\begin{array}{c}\text { Total } \\
\mathrm{n}=35\end{array}$ & \\
\hline Parcial & 26 & 5 & 31 & \\
Generalizada & 4 & 0 & 4 & $\mathrm{p}=0,5234$ \\
Precoce & 4 & 0 & 4 & \\
Tardia & 26 & 5 & 31 & $\mathrm{P}=0,5234$ \\
\hline
\end{tabular}

As idades das pacientes do sexo feminino que apresentaram crises generalizadas (média=82,5 anos) foi significantemente ( $p<0,05$ ) maior do que aqueles com crises parciais (média $=57,2$ anos). No sexo masculino, pacientes com crises generalizadas tiveram média de idades de 43,0 anos e com crises parciais de 55,8 anos, sem diferença significante.

Nāo houve associação entre o tipo de crise e o intervalo de tempo para seu aparecimento em relação ao AVCI.

A frequência das crises não se associou nem ao tipo de crise, nem ao intervalo de tempo para seu aparecimento após o AVCI (Tabela 1).

Os resultados da análise das correlações entre o tipo de crise (parcial ou generalizada) e antecedentes pessoais de hipertensão arterial, diabetes melito, AVC pregresso, AIT e etilismo podem ser observadas na Tabela 2. O tipo de crise não se associou aos antecedentes estudados, exceto as crises parciais que se associaram a tabagismo $\left(\mathrm{p}=0,03^{*}\right)$.

Os resultados da análise das correlações entre crise epiléptica precoce $e$ tardia com antecedentes pessoais de hipertensão arterial, diabetes melito, AVC pregresso, ataque isquêmico transitório, tabagismo e etilismo são apresentados na Tabela 3.

A alteração do eletrencefalograma (EEG) predominante foi a de alentecimento focal da atividade elétrica cerebral $(56,8 \%)$, seguida por alentecimento difuso $(10,8 \%)$ e atividade paroxística anormal $(8,1 \%)$, sendo que 2 pacientes apresentaram mais que uma anormalidade. O EEG foi normal em $24,3 \%$ dos pacientes.

Tabela 2. Pacientes com AVCI e crises epilépticas segundo o tipo de crise parcial e generalizado e segundo a presenf̧a de antecedentes pessoais relacionados ao AVC. Resultado do teste exato de Fisher.

\begin{tabular}{lccc}
\hline & $\begin{array}{c}\text { Parcial } \\
\mathrm{n}=31\end{array}$ & $\begin{array}{c}\text { Generalizada } \\
\mathrm{n}=4\end{array}$ & $\mathrm{p}$ \\
\hline Hipertensão arterial & 13 & 1 & 0,4699 \\
Diabetes melito & 3 & 1 & 0,3991 \\
AVC pregresso & 14 & 1 & 0,4191 \\
AIT & 9 & 1 & 0,6809 \\
Tabagismo & 19 & 0 & $0,0345^{*}$ \\
Etilismo & 8 & 1 & 0,7324 \\
\hline
\end{tabular}


Tabela 3. Pacientes com AVCI e crises epilépticas segundo tempo de aparecimento das crises epilépticas: precoce ( 1 -14 dias) e tardia (>14 dias) e segundo a presenfa de antecedentes pessoais relacionados ao AVC. Resultado do teste exato de Fisher.

\begin{tabular}{lccc}
\hline & $\begin{array}{c}\text { Precoce } \\
\mathrm{n}=4\end{array}$ & $\begin{array}{c}\text { Tardia } \\
\mathrm{n}=31\end{array}$ & $\mathrm{p}$ \\
\hline Hipertensão arterial & 2 & 13 & 0,5809 \\
Diabetes melito & 0 & 4 & 0,6009 \\
AVC pregresso & 1 & 15 & 0,3701 \\
AIT & 1 & 10 & 0,6282 \\
Tabagismo & 3 & 17 & 0,4191 \\
Etilismo & 1 & 8 & 0,7324 \\
\hline
\end{tabular}

Apenas 7 pacientes tiveram a oportunidade de realizar EEG antes da primeira crise epiléptica, e a distribuição das anormalidades estudadas comportou-se de maneira semelhante aos dos pacientes que realizaram o exame após a crise.

Não houve associação entre tipo de crise e tamanho da lesão $(p=0,57)$, e nem do tipo de crise e numero de lesões $(p=0,53)$.

Todos os três pacientes com estado de mal epiléptico apresentaram alterações do EEG, observando-se atividade paroxistica anormal e alentecimento focal no primeiro, somente alentecimento focal no segundo e somente alentecimento difuso no último. Quanto á tomografia, o primeiro apresentou lesāo única nāo extensa parietal esquerda, com atrofia cerebral; os dois outros apresentaram lesões múltiplas em ambos hemisférios.

Os resultados do teste de Mann-Whitney mostraram que o intervalo de tempo entre o AVCI e a primeira crise epiléptica não se associou ao número de lesōes (Zcalculado $=0,07 ; Z c r i ́ t i c o=1,96$ ), nem ao tamanho da lesão (Zcalculado=0,82; Zcrítico=1,96).

Os pacientes foram tratados com fenobarbital (18 pacientes) ou com fenitoína (16 pacientes). Um paciente não recebeu droga anti-epiléptica. Todos obtiveram controle satisfatório das crises.

\section{DISCUSSÄO}

Os aspectos clínicos e laboratoriais referentes aos pacientes deste estudo foram comparados aos de um grupo controle de pacientes com AVCI que evoluíram sem crises epilépticas e foram abordados anteriormente $e^{21,23,24}$. Chamou-nos a atenção a associação entre tabagismo e etilismo ao grupo de epilépticos ${ }^{23}$; e dentre os exames laboratoriais, destacaram-se quanto à preditividade das crișes epilépticas após AVCl, o conjunto de presença de lesão extensa à TC e alteração do EEG (principalmente do tipo alentecimento focal) ${ }^{24}$.

Observamos que $11 \%$ das crises ocorreram no período de 1 a 14 dias, tendo sido excluídos pacientes que apresentaram crise apenas nas primeiras 24 horas após a instalação do AVCI, que poderiam ter sido causadas por distúrbios metabólicos, hemodinâmicos, edema cerebral, abstinência alcoólica, entre outras causas, e, acreditamos, constituem um grupo com caracteristicas diferentes do grupo estudado; $34 \%$ dos pacientes apresentaram crise no período de 15 a 180 dias; $54 \%$ apos 180 dias, sendo que somente $17 \%$ dos pacientes apresentou crise após 1 ano.

A maioria dos estudos publicados ${ }^{2,3,27,29,32,45}$ classifica as crises epilépticas segundo o tempo entre o AVC e a crise em: crises precoces, até 2 semanas após o AVC; crises tardias, após 2 semanas do AVC. 
A diferença entre crise precoce e tardia recai basicamente sobre o mecanismo fisiopatológico nos casos de epilepsia ${ }^{34}$. As crises precoces parecem resultar das reaçōes bioquímicas provocadas pela lesão aguda no cérebro, caracterizadas por isquemia neuronal, edema e acúmulo de substâncias citotóxicas ou metabólitos; enquanto as crises tardias se relacionam à formação de cicatriz por gliose, causando focos epilépticos crônicos ${ }^{10,27,29,45}$. Crise epiléptica pode ser a primeira manifestação de um AVC ${ }^{2,32,40}$, tendo sido denominada "epilepsia irritativa". De Reuck et al. ${ }^{14}$, porém apontaram que, na maioria desses casos, pode haver outras causas para a crise epiléptica, que não o AVC, por exemplo, insuficiência renal, hipertensão arterial, edema cerebral, hipertensão intracraniana, e outros fatores hemodinâmicos, que podem desencadear crises epilépticas com início numa área previamente infartada. A maior incidência de crises epilépticas, porém, ocorre no período de 6 a 12 meses após o AVC $^{18.48}$. Alguns autores, no entanto, descreveram a ocorrência de crise epiléptica após AVC em 2 picos de frequência, o primeiro nas duas primeiras semanas e outro entre 6 meses e 1 ano após a instalação do $\mathrm{AVC}^{5.25 .32 .45}$.

Observamos nos nossos pacientes, $89 \%$ de crises do tipo parcial e $11 \%$ do tipo generalizado; este resultado é bastante parecido aos obtidos por vários autores; encontramos apenas um paciente com crise parcial complexa. Winckler ${ }^{52}$, no Brasil, observou crises parciais com generalização subsequente em $57 \%$, parciais complexas em $7 \%$, parciais simples em $7 \%$ e generalizadas em $29 \%$ dos 14 casos de epilepsia de início tardio cuja causa foi doença vascular cerebral. $\mathrm{O}$ tipo de crise mais frequentemente descrito é o tipo parcial, e dentre as crises parciais, a parcial motora com ou sem generalizaçāo subsequente tem sido destacada ${ }^{9,31.32}$; tenta-se explicar este fato argumentando que os vasos que suprem a região rolândica sejam mais propensos a oclusão, ou que o limiar para crises parciais motoras seja menor que o de outros tipos de crises ${ }^{29}$. Sung e $\mathrm{Chu}^{45}$ relatam $56 \%$ de crises do tipo parcial simples, $24 \%$ de crises do tipo parcial complexa, $4 \%$ do tipo generalizado e $16 \%$ de crises inclassificáveis, em uma das maiores casuísticas de crises epilépticas secundárias a AVCI do tipo trombótico. Os achados de Cocito et al. ${ }^{9}$ a respeito do tipo de crise sāo semelhantes aos encontrados pelos autores acima, isto é, $82 \%$ de crises do tipo parcial e $18 \%$ do tipo generalizado. Gupta et al. ${ }^{25}$, discordando, relataram $58 \%$ de crises do tipo generalizado, contra $42 \%$ do tipo parcial, em estudo de 90 pacientes com AVCI.

A presença de episódio de estado de mal epiléptico em $9 \%$ dos pacientes da nossa amostra parece assemelhar-se à frequência relatada pela maioria dos autores ${ }^{31}$. Apesar do estado de mal epiléptico nāo ser frequente nos AVC, estes constituem a segunda causa de estado de mal epiléptico sintomático agudo, isto é, secundário a condição aguda, só superados por distúrbios metabólicos (uremia, hiperglicemia, insuficiência hepática, distúrbios eletrolíticos ou causas mistas). Os AVC são também a segunda causa de estado de mal epiléptico sintomático remoto, isto é, com história pregressa de lesão cerebral, só superados neste caso, por trauma cranioencefálico ${ }^{4}$. Em estudos sobre estado de mal epiléptico em pacientes com mais que 60 anos, os AVC são considerados a principal etiologia, chegando a constituir $35 \%$ dos $\operatorname{casos}^{45}$. Sung e $\mathrm{Chu}^{45}$ relataram que $13 \%$ dos 118 pacientes com AVCI apresentaram mal epiléptico, sendo que $80 \%$ deles após a fase aguda. Gupta et al. ${ }^{25}$ encontraram estado de mal epiléptico em $8 \%$ dos 90 pacientes com AVCI. Há, no entanto, relatos discrepantes, como por exemplo o de Cocito et al. ${ }^{9}$ que, estudando 22 casos de oclusão da artéria carótida ou da cerebral média, encontraram estado de mal epiléptico em 8 (36\%). Barolin ${ }^{2}$ relacionou estado de mal epiléptico com mau prognóstico do AVC quanto a presença de sequelas motoras, porque acredita que um cérebro com doença cerebrovascular raramente seja capaz de sustentar descargas adicionais de sintomas epilépticos contínuos, levando à ampliação da área lesada.

Concluindo, as crises epilépticas após AVCI são na maioria dos casos do tipo parcial e iniciamse após a fase aguda, com pico de frequência no período de 6 meses a 1 ano após a instalação do AVCI; podem ser consideradas benignas, pois poucas vezes produzem estado de mal epiléptico e a recorrência é pouco frequente. O controle medicamentoso é facilmente obtido com monoterapia. 
Agradecimentos - Agradecemos ao Prof. Dr. José Geraldo de Camargo Lima pela orientação da tese que originou este estudo, aos Prof. Dr. Neil Ferreira Novo e Profa. Dra. Yara Juliano pela orientação da análise estatística e ao Prof. Dr. Carlos José Reis de Campos pelas correçōes, críticas e sugestōes.

\section{REFERÊNCIAS}

1. Annegers JF, Hauser WA, Lee JR, Rocca WA. Incidence of acute seizures in Rochester, Minnesota 1935-1984. Epilepsia 1995;36:327-333.

2. Barolin GS. The cerebrovascular epilepsies. Eletrencephalogr Clin Neurophysiol 1982;35 (Suppl.):287- 295.

3. Barolin GS, Scherz A. The cerebrovascular epilepsies. Eletrencephalogr Clin Neurophysiol 1974;27:347-352.

4. Barry E, Hauser WA. Status epilepricus: the interaction of epilepsy and acute brain disease. Neurology 1993;43:1473-1478.

5. Blandin CF, Johnston PJ, Norris JW. Stroke Research Unit, University of Toronto, Canada for The Seizures after Stroke Study Group (Canada, Australia, Israel, Italy). Stroke 1993;24:49.

6. Browne TR, Feldman RG. Epilepsy: an overview. In: Browne TR, Feldman RG. Epilepsy: diagnosis and management. Boston: Little, Brown, 1983:1-10.

7. Celesia GG. Modern concepts of status epilepticus. JAMA 1971:235:1571-1574.

8. Chusid JG, Kopeloff LM. Epileptogenic effects of pure metals implanted in motor cortex of monkeys. J Appl Physiol 1962:17:696-700.

9. Cocito L, Favale E, Reni L. Epileptic seizures in cerebral arterial occlusive disease. Stroke 1982;13:189-195.

10. Cocito L, Favale E, Reni L. The frequency, characteristics and prognosis of epileptic seizure at the onset of stroke. J Neurol Neurosurg Psychiatry 1989;52:292.

11. Commission Of Classification And Terminology Of The International League Against Epilepsy. Proposal for revised clinical and eletroencephalografic classification of epileptic seizures. Epilepsia 1981;22:489-501.

12. Commission of Classification and Terminology of The International League Against Epilepsy. Proposal for revised classification on epilepsies and epileptic syndromes. Epilepsia 1989;30:389-399.

13. Dams AM, Fuglsang-Frederickson A, Svarre-Olsen U, Dam M. Late onset epilepsy, etiologies, types of seizure and value of clinical investigation, EEG and CT scan. Epilepsia 1986:26:227-231.

14. DeReuck J, Krahel N, Sieben G, Orban L, Decoster W, VanderEcken H. Epilepsy in patients with cerebral infarcts. J Neurol 1980;224:101-109.

15. Ettinger AB. Structural causes of epilepsy: tumors, cysts, stroke, and vascular malformations. Neurol Clin 1994;12:41-56.

16. Farnarier G, Iemolo F. Etude longitudinale des epilepsies survenant chez l'adulte. Rev EEG Neurophisiol 1981:11:531-536.

17. Fentz V. Epileptiske anfaldsfaenomener hos patienter med apoplexia cerebri. Nord Med 1971;86:1023-1025.

18. Fish DR, Miller DR, Roberts RC, Blackie JD, Gilliatt RW. The natural history of late-onset epilepsy secondary to vascular disease. Acta Neurol Scand 1989;80:524-526.

19. Forsgren L, Bucht $G$ Ericksson S,Bergmark L. Incidence and clinical characterization of unprovoked seizures in adults: a prospective population-based study. Epilepsia 1996;37:224-229.

20. Fuerstein J, Weber M, Kurtz D, Rohmer F. Etude statistique des crises épileptiques apparaisant apres 1'âge de 60 ans. Semin Hôp Paris 1970;46:3125-3128.

21. Fukujima MM. Crises epilépticas após acidente vascular cerebral por infarto cerebral: Estudo de 35 pacientes. Tese de Mestrado, Escola Paulista de Medicina, Universidade Federal de São Paulo. São Paulo, 1994.

22. Fukujima MM. Epileptic seizures after ischemic stroke: study of 35 patients. (Abstract). Arq Neuropsiquiatr 1996;54:714.

23. Fukujima MM, Cardeal JO, Lima JGC. Estudo de fatores clínicos preditivos para crises epilépticas após acidente vascular cerebral isquêmico. Arq Neuropsiquiatr 1996;54:207-211.

24. Fukujima MM, Cardeal JO. Exames subsidiários preditivos de crises epilépticas após acidente vascular cerebral isquêmico. Arq Neuropsiquiatr 1997;55:39-45.

25. Gupta SR, Naheedy MH, Elias D, Rubino FA. Posuinfarction seizures: a clinical study. Stroke 1988;19:1477-1481.

26. Hauser WA, Kurland LT. The epidemiology of epilepsy in Rochester, Minnesota, 1953 though 1967. Epilepsia 1975;16:1-66.

27. Heiss WD, Hayakama T, Waltz AG. Cortical neuronal function during ischemia. Arch Neurol 1976;33:813-820.

28. Janz D, Masuhr KF. Epilepsia tarda: a clinical, EEG and neurorradiological study (taking in consideration cranial computerized tomography). Epilepsia 1978;19: 110.

29. Lesser R, Luders H, Dinner DS, Morris HH. Epileptic seizures due to thrombotic and embolic cerebrovascular disease in older patients. Epilepsia 1985;26:622-630.

30. Levitt P, Wilson WP, Wildins RH. The effects of subarachnoid blood on the electrocorticogram of the cat. J Neurosurg 1971;35:185-191.

31. Lo YK, Yiu CH, Hu HH, Su MS, Laeuchli SC. Frequency and characteristics of early seizures in Chinese acute stroke. Acta Neurol Scand 1994;90:83-85.

32. Louis S, McDowell F. Epileptic seizures in nonembolic cerebral infarction. Arch Neurol 1967:17:414-418.

33. Luhdorf F, Jensen LK, Plesner A.Etiology of seizures in the eldery. Epilepsia 1986;27:458-463.

34. Marosi M, Luef G, Schett P, Graf M, Sailer U, Bauer G. The effects of brain lesions on the course os chronic epilepsies. Epilepsy Res 1994;19:63-69.

35. Merlis JK. Epilepsy of late onset. In Magnus O, Haas AML.The epilepsies. New York: Elsevier, 1974:264-270.

36. Mohr JP, Caplan LR, Melski JW, Goldstein RJ, Duncan GW, Kistler JP, Pessin MS, Bleich HL. The Harvard cooperative stroke registry: a prospective registry. Neurology 1978;28: 754-762.

37. Moskowitz E, Lightbody FEH, Freitag NS. Long-term follow-up of the poststroke patient. Arch Phys Med Rehab 1972;53:167-172. 
38. Olsen TS, Hogenhaven H, Thage O. Epilepsy after stroke. Neurology 1987;37:1209-1211.

39. Penfield W, Jasper H. Epilepsy and functional anatomy of the human brain. Boston: Little, Brown \& Co., 1954.

40. Richardson EP, Dodge PR. Epilepsy in cerebral vascular disease: a study of the incidence and nature of 104 consecutive proven cases of cerebral infarction and hemorhage. Epilepsia 1954;3:49-74.

41. Schold C, Yarnell PR, Earnest MP. Origin of seizures in eldery patients. JAMA 1977;238:1177-1178.

42. Shinton RA, Gill JS, Melnick SC, Gupta AK, Beevers DG. The frequency, characteristics and prognosis of epileptic seizure at the onset of stroke. J Neurol Neurosurg Psychiatry 1988;51:273-276.

43. Siegel S. Estadistica no parametrica. México: Trillas, 1975:346.

44. So EL, Annegers JF, Hauser WA, O'Brien PC, Whisnant JP. Population-based study of seizure disorders after cerebral infarction. Neurology 1996;46:350-355.

45. Sung CY, Chu NS. Epileptic seizures in thrombotic stroke. J Neurol 1990;237:166-170.

46. Vasconcelos D, Lombardo L. Crisis epilépticas del adulto: estudio prospectivo de 65 casos. Gac Med Mex 1983;1 19:23-27.

47. Vercelletto P, Delobel-Cozan $R$. Étude des facteurs étiologiques et prognostiques dans les Epilepsies débutant après 60 ans. Semin Hôp Paris 1970;46:3133-3137.

48. Vitaren M, Erickson S, Asplund K. Risk of recurrent stroke, myocardial infarction and epilepsy during long-term followup after stroke. Eur Neurol 1988;28:227-231.

49. Whisnant JP, Basford JR, Bernstein EF, et al. Special report from the National Institute of Neurological Disorders and Stroke. Classification of Cerebrovascular Disease III. Stroke 1990;21:637-676.

50. White PT, Bailey AA, Bickford RG. Epileptic disorders in the aged. Neurology 1953;3:674-679.

51. Willmore LJ, Herd RW, Sypert GW. Epileptiform activity initiated by pial iontophoresis of ferrous and ferric chlorid on rat cerebral cortex. Brain Res 1978;152:406-410.

52. Winckler TCD. Crises epilépticas de início tardio: análise de 100 casos. Tese de Mestrado, Escola Paulista de Medicina. Sảo Paulo, 1988.

53. Woodcock S, Cosgrove JBR. Epilepsy after the age of 50: a five-year follow-up study. Neurology 1964;14:34-40. 